\title{
Design considerations to improve cognitive ergonomic issues of unmanned vehicle interfaces utilizing video game controllers
}

\author{
P. Oppold; M. Rupp; M. Mouloua*; P.A. Hancock; and J. Martin \\ Transportation Research Group, Psychology Department, University of Central Florida, 4000 Central Florida \\ Blvd. Orlando, FL 32816-1390, USA
}

\begin{abstract}
Unmanned (UAVs, UCAVs, and UGVs) systems still have major human factors and ergonomic challenges related to the effective design of their control interface systems, crucial to their efficient operation, maintenance, and safety. Unmanned system interfaces with a human centered approach promote intuitive interfaces that are easier to learn, and reduce human errors and other cognitive ergonomic issues with interface design. Automation has shifted workload from physical to cognitive, thus control interfaces for unmanned systems need to reduce mental workload on the operators and facilitate the interaction between vehicle and operator. Two-handed video game controllers provide wide usability within the overall population, prior exposure for new operators, and a variety of interface complexity levels to match the complexity level of the task and reduce cognitive load. This paper categorizes and provides taxonomy for 121 haptic interfaces from the entertainment industry that can be utilized as control interfaces for unmanned systems. Five categories of controllers were based on the complexity of the buttons, control pads, joysticks, and switches on the controller. This allows the selection of the level of complexity needed for a specific task without creating an entirely new design or utilizing an overly complex design.
\end{abstract}

Keywords: Cognitive-Load; Tele-Operation; Human Computer Interaction

\section{Introduction}

The use of unmanned (UAVs, UCAVs, and UGVs) systems are seen in search and rescue, combat operation, surveillance, and several other associated realms. However, there are still some major human factors and ergonomic challenges related to the effective design of their control interface systems. For example, as of 2004, the accident rates of unmanned systems (US) were 10 times greater than similar manned aircraft systems. Consequently, 70$80 \%$ of these accidents were due to cognitiveergonomic issues related to control interface of the US [10]. Having effective control interfaces and design guidelines is crucial for the efficient operation, maintenance, and safety of these systems. Similarly, poorly designed control interfaces may also increase the cognitive workload and decrease operator's per- formance [4,6,11]. Thus, designing US interfaces with a human centered approach would promote an easier to learn intuitive interface, reducing human errors, free up mental resources, and reduce workload to improve performance [6-7].

The current solution to this issue has been to increase automation, which shifted the workload from physical to cognitive. Due to this workload shift, control interfaces used for US need to evolve alongside the tasks of piloting the vehicles [2,5]. Control interfaces for US should be designed to reduce mental workload on the operators whenever possible and facilitate the interaction between vehicle and operator. Interfaces that are not designed to decrease cognitive workload on the operator in this manner lead to unavoidable errors $[5,7]$.

\footnotetext{
* Corresponding author. E-mail: Mustapha.Mouloua@ucf.edu. Tel: 407-823-2910.
} 
Two-handed video game controllers are an interface format previously accessible to many new UV operators [1,11]. These user interfaces for US are effective design considerations for systems with some level of task automation, where the user must stay within the control loop. These commercial off the shelf devices provides wide usability within the overall population, prior exposure for new operators, and an interface that can be customized to the level of complexity of the task [1]. This leads to an interface that is better designed for that particular task to reduce cognitive interface load, instead of a one-sizefits-all approach which ignores a true understanding of the task complexity [3].

Game controllers have undergone many competing design changes over time, similar to other US interfaces such as keyboards. Multiple designs have tried to optimize the overall design by centering on a detailed understanding of the user experience, with performance considerations leading to user-centered and task centered approaches and new designs [3]. The recent iteration of this process, Playstation 3 and Xbox controllers, are being utilized for unmanned system interfaces [1,11]. Prior controllers were designed the same way, and have similar merits for usage consideration.

\section{Methods}

This paper categorizes and provides taxonomy of 121 haptic interfaces released $1972-2010$ by the entertainment industry. Controllers selected for this analysis were used by more than one game, were separate units from the console via wireless or a cord, could be verified for the variable values, were operated with two hands consistently on the controller, and were commercially released as a product [8]. First and third party reverse compatible controllers were used, but controllers that were clones of existing products were only counted once based on their original release format. Duplicated or redundant features were not included, nor were features that could not be operated while both hands were holding the controller.

Controllers have four interface variables: buttons, control pads, switches and joysticks. Each interface variable includes the evolution of the technology format over time, each successive iteration of the technology provided additional degrees of freedom for movement, operation, or selection processes. Thus the control pad variable covers the evolution of the technology format from dials and knobs to control discs and then later to direction pads, all used for the same input process on the controller.

\section{Results and Discussion}

Controllers were placed into two different five category configurations first based on internal consistency (fig. 1) and secondly chronologically (fig. 2).

Internal and External consistencies were used as the organizing principle for the first configuration by examining the frequency and placement of input variables [3]. These categories (fig. 1) represent a spread of competing designs, similar to how UV interfaces that use keyboards have access to several valid alternative designs [3].

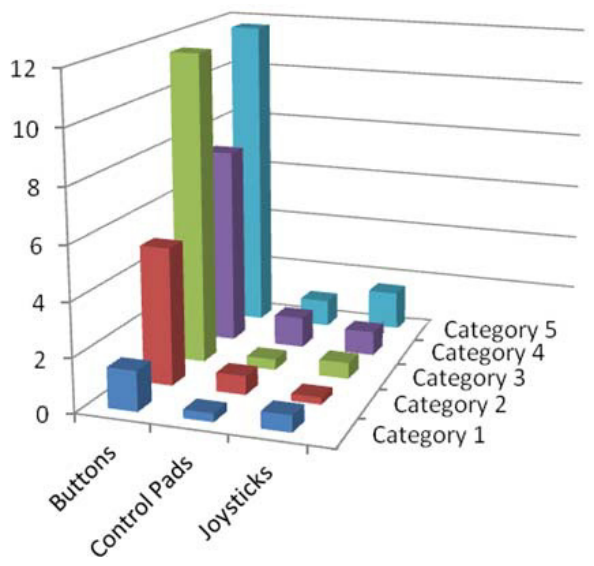

Fig 1: Categories by Internal and External Consistency

Secondly, the controllers were grouped into an additional five categories based on chronological development cycles of nine years over the entire range of the dataset (1972-1978, 1979-1988, 1989-1998, 1999-2008, \& 2008-2010). Over time the variable means have increased, with the mean for buttons showing the largest increase (fig. 2). This demonstrates the increase of complexity in the controller designs over time, with newer designs being more complex than prior designs. 


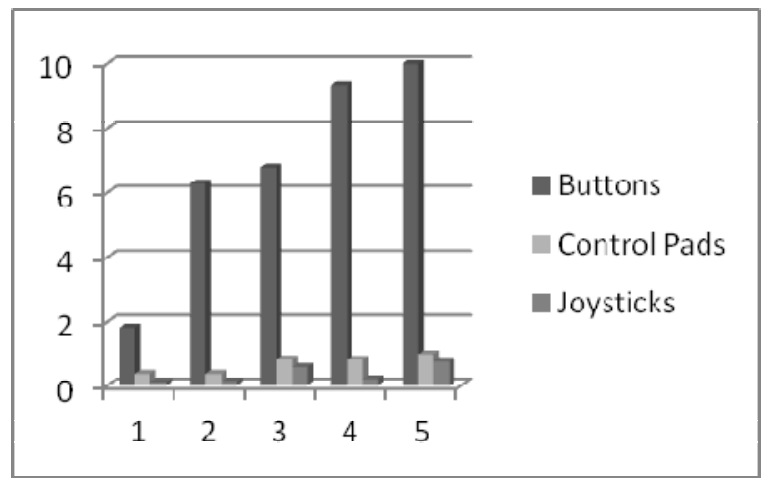

Fig 2: Overall means of categories by 9 year interval

The overlap between the increase of design complexity over time and the development of alternative designs, which remain valid for contemporary utilization, shows that older controllers are less valid than less complex alternative designs. Older controllers provide less complex interfaces than newer interfaces do which may represent loss of functions.

\section{Recommendations}

Categorizing controllers based on chronology diminishes the usefulness older designs may have [3]. Older controller designs should be evaluated for use in interface design for US based on complexity of alternate designs. Tasks should be evaluated in regards to interface complexity and user needs so that the correct level of interface complexity needed for a US specific task can then be determined. This will provide US interfaces to varying levels of task complexity without creating an entirely new design or utilizing one that is too complex for the task and will increase cognitive workload $[3,7]$.

This process leads to an interface that is better designed for that particular task and not a one-size-fitsall approach which ignores a true understanding of the task and the user [3]. The categories of controllers share external consistency, facilitating users to utilize their already formed mental model of the current category 5 controllers in use (Playstation 3, Xbox 360) in order to reduce training time and workload on prior categories of controllers [3,9].

Controller design categories provide a framework of user-centered evaluation of commercially available off-the-shelf controller designs, which can be used for commercial, military, and academic research and applications. This will assist in determining the appropriate controllers, past and present, for research, search and rescue, military, bomb disposal and emergency response applications based on their level of interface complexity and the complexity of the task $[2,9]$.

These recommendations would move the usage of video game controllers as US interfaces from only the most recent designs to the usage of several categories of alternative designs utilizing varying complexity exhibited over 30 years of iterative development [9].

\section{References}

[1] D. Axe \& S. Olexa, War Bots: How U.S. Military Robots Are Transforming War in Iraq, Afghanistan, and the Future. Ann Arbor, MI: Nimble Books, 2008.

[2] T. Fong, \& C. Thorpe. Vehicle Teleoperation Interfaces. Autonomous Robots (11), (2001), 9-18.

[3] J. Grudin. The Case Against User Interface Consistency. Human Aspects of Computing, 32 (10), (1989), 1164-1173.

[4] P.A. Hancock, M. Mouloua, R. Gilson, J. Szalma, \& T. OronGilad. Provocation: Is the UAV Control Ratio the Right Question? Ergonomics in Design, Winter, 7-31, 2007.

[5] J. McCarley, \& C. Wickens. Human Factors Implications of UAVs in the National Airspace (pp. 1-63): Institute of Aviation Aviation Human Factors Division University of Illinois at Urbana-Champaign, 2004.

[6] M. Mouloua, R. Gilson \& P. Hancock. Human-Centered Design of unmanned Aerial Vehicles. Ergonomics in Design, Winter, (2003), 6-11.

[7] S. Oviatt. Human-Centered Design Meets Cognitive Load Theory: Designing Interfaces that Help People Think. Paper presented at the ACM MM 2006, Santa Barbara, CA (2006).

[8] P. Oppold, M. Rupp, M. Mouloua \& J. Martin. [Trend Analysis of two-handed video game controllers]. Unpublished raw data, 2011.

[9] R. Stahl. Have you played the war on terror? Critical studies in media communication, 23 (2), (2006), 112-130.

[10] K. Williams. A Summary of Unmanned Aircraft Accident/Incident Data: Human Factors Implications (pp. 1-18) Oklahoma City, OK: Civil Aerospace Medical Institute Federal Aviation Administration, 2004.

[11] D. Voth. A New Generation of Military Robots. IEEE Intelligent Systems, 8, (2008) 1-3. 